\title{
Introduction to the Digital Mobile Services for Everyday Life Mini-Track
}

\author{
Pirkko Walden \\ Institute for Advanced Management \\ Systems Research (IAMSR), Turku, \\ Finland \\ pirkko.walden@abo.fi
}

\author{
Tomi Dahlberg \\ Turku School of Economics \\ at University of Turku \\ Turku, Finland \\ tomi.dahlberg@utu.fi
}

\author{
Anna Sell \\ Åbo Akademi University \\ Turku, Finland \\ anna.sell@abo.fi
}

Digital mobile services have become integral parts of our everyday lives, enhancing and changing many of our activities and routines. Everyday life is highly personal; while certain activities must be performed everyday by everyone - such as sleeping and eating - there is limitless variation in how, when and where individuals choose to do these and the myriad of other mundane and more complex activities. Despite the endless multiplicity of our days, digital mobile services are our constant companions. They assist in navigating manifold social contexts daily, through shifting physical locations and various degrees of mobility and with both physical and mental activities. Many everyday activities are repeated or routines, making them especially amenable to smart support and support for behavior change through advances in sensor technologies and data analytics.

Health and wellness is an area of everyday life garnering ample interest from both the technology industry, research community and society. At the same time, a rapidly aging population structure in many countries pushes public health systems to seek cost-effective ways to support healthy aging. While wellness technologies have rapidly gained users, there are many unanswered or unexplored questions regarding the potential of technology to aid in forming beneficial, lasting wellness routines, in changing behavior patterns, and in influencing future wellbeing. Older adults' technology use is another quandary only partially understood. How elderly individuals make use of technology is also a moving target - the proliferation of technology in working life means that adults entering retirement are increasingly technology-savvy when compared to previous generations, rendering many of our previous conclusions about elderly technology users less relevant or outright outdated.

Information systems science researchers play an important role in advancing our understanding about the role(s) of digital mobile services in everyday life, including at the intersection of aging and wellness. We need to build on the knowledge and theories within this research area to offer theoretical explanations and to provide guidance to the users, developers, and regulators of mobile services. The Digital Mobile Services for Everyday Life mini-track has since its very start in 2002 URI: https://hdl.handle.net/10125/79510

978-0-9981331-5-7

(CC BY-NC-ND 4.0) been an arena for innovative research contributions that open up new perspectives and insights to better deploy and use mobile technologies, applications and services.

Research in numerous everyday contexts of mobile services calls for methodological diversity and creativity. We are pleased to note this to be reflected in the papers submitted to this year's mini-track. The three papers accepted after extensive peer reviews and one round of revisions address digital wellness services for elderly people, contributing to this topical and extremely important research issue in most societies.

The first paper Survival of the Fittest? Examining Lapsing Behaviour in the Context of Elderly People and the Use of Physical Activity Tracker Applications, by Makkonen et al. found that lapsing behaviour is affected mainly by the initial physical activity level as well as partly by gender and age but not by household type and only weakly by technology readiness.

The second paper Does Physical Activity Application Use Promote Self-Efficacy for Exercise? A Study Among Aged People by Kari et al. compared self-efficacy levels between three different time points: before taking the application into use and after 4 and 12 months of use. Their results suggest that physical activity application use can be effective in promoting self-efficacy for exercise among aged people as there was an improvement in most of the self-efficacy items as well as in total self-efficacy already after 4 months of use and this improvement was sustained after 12 months of use.

The third paper Technology readiness, UTAUT2 and continued use of digital wellness services - A configurational approach by Mezei et al. found that technology readiness can contribute to our understanding of users' continued usage behavior, both as an independent dimension and when combined with some of the UTAUT2 dimensions. The approach is novel, as TR and UTAUT2 have not previously been used in combination and analyzed with the Fuzzy-set Qualitative Comparative Analysis (FsQCA) methodology. Using FsQCA, we can better capture and understand complex and even paradoxical attitudes towards technology. 